\title{
A COUNTABLE SELF-INJECTIVE RING IS QUASI-FROBENIUS ${ }^{1}$
}

\author{
JOHN LAWRENCE
}

ABSTRACT.A countable dimensional self-injective algebra is Artinian. There is an application to self-injective twisted group algebras.

It has been known for some time that a countable self-injective ring is semilocal (see for example [8]). In this paper we show that such a ring is in fact quasi-Frobenius. Special cases of this result have been proved previously, for example if the ring is also regular [3] or if it is a group algebra [8]. My thanks to Ken Louden for his help in the preparation of this paper.

Unless stated otherwise, all rings are associative with a unity. If $S$ is a subset of a ring $R$, we denote its left annihilator in $R$ by $l_{R}(S)$.

TheOREM 1 (FAITH [1]). A ring is quasi-Frobenius if it is right self-injective and satisfies the descending chain condition on left annihilators.

Proposition 2. Let $R$ be a subring of $S$. Suppose that $S_{S}$ is injective, ${ }_{R} S$ is flat and $S_{R}$ is free. Then $R_{R}$ is injective.

Proof. The proof is left to the reader.

THEOREM 3. Every countable subring of a quasi-Frobenius ring is contained in a countable quasi-Frobenius subring. Conversely, if every countable subring of a ring is contained in a quasi-Frobenius subring, then the ring is quasi-Frobenius.

Proof. Suppose first that $T$ is a quasi-Frobenius ring and $A$ is a countable subring. We construct a sequence of subrings $A=R_{0} \subset R_{1} \subset R_{2} \subset \cdots \subset$ $T$ inductively as follows. Given $R_{k}$, consider all $n$-tuples $\left\{a_{1}, \ldots, a_{n}\right\}$ of elements of $R_{k}$ as $n$ ranges over the positive integers. If $a_{n} \in a_{1} T+\cdots+$ $a_{n-1} T$ choose $x_{1}, x_{2}, \ldots, x_{n-1} \in T$ so that $a_{n}=a_{1} x_{1}+\cdots+a_{n-1} x_{n-1}$. If $a_{n} \notin a_{1} T+\cdots+a_{n-1} T$, choose $x_{n} \in T$ so that $x_{n} a_{i}=0, i=1,2, \ldots, n$ -1 , and $x_{n} a_{n} \neq 0$. Now do the same for the left ideal generated by $a_{1}, a_{2}, \ldots, a_{n-1}$. Let $R_{k+1}$ be the subring of $T$ generated by $R_{k}$ and all the $x$ 's obtained. Let $R=\cup_{i=1}^{\infty} R_{i}$. Clearly $A \subset R$ and $R$ is a countable subring, so

Received by the editors June 23, 1976.

AMS (MOS) subject classifications (1970). Primary 16A52; Secondary 16A26.

Key words and phrases. Injective ring, group algebra.

'This paper was written while the author was a member of the Summer Research Institute of the Canadian Mathematical Congress at Dalhousie University.

- American Mathematical Society 1977 
we need only show that $R$ is quasi-Frobenius.

As $T$ is right and left Artinian, $R$ satisfies $A C C$ and $D C C$ on right and left annihilators. If $I$ is a finitely generated right ideal of $R$, then, by construction, $r_{R}\left(l_{R}(I)\right)=I$. A dual result holds for left ideals. Thus $R$ satisfies $A C C$ on finitely generated right and left ideals and so is right and left Noetherian. As $R$ satisfies $D C C$ on right and left annihilators it is right and left Artinian. Since $R$ is right and left Artinian and satisfies the 'annihilator condition' [9, p. 276], $R$ is quasi-Frobenius.

Now suppose that every countable subring of $T$ is contained in a quasiFrobenius subring. Then $T$ is clearly right and left Artinian. In order to prove that $T$ is right self-injective, we need only show that for all finitely generated right ideals $I$ and $J$ we have $r(l(I))=I$ and $l(I \cap J)=l(I)+l(J)$, (see $[9$, p. 274]). However, if these conditions were not satisfied we could construct a countable subring $A$ such that for any subring between $A$ and $T$ these would not be satisfied, and this contradicts the hypothesis that $A$ is a subring of a quasi-Frobenius ring.

THEOREM 4. Let $R$ be a right self-injective ring and let $\left\{J_{i}\right\}_{i \in \Psi}$ be a descending chain of left annihilators, well-ordered by inclusion. Then the cardinality of $\Psi$ is less than the cardinality of $R$.

Proof of THE THeOREM. Suppose that the cardinality of $\Psi$ is greater than or equal to the cardinality of $R$. We may suppose that $\Psi$ is a set of ordinals. Let $\Phi$ be the set of ordinals strictly less than the cardinality of $\Psi$, thus $|\Phi|=|R|$, and we consider the descending chain of annihilators $\left\{J_{i}\right\}_{i \in \Phi}$. Suppose $R=\left\{a_{j}\right\}_{j \in \Phi}$. Suppose $J_{i}$ annihilates the right ideal $I_{i}$ on the left. Let $J=\bigcap_{i \in \Phi} J_{i}$ and let $I=\cup_{i \in \Phi} I_{i}$. Clearly $J$ is the left annihilator of $I$. Consider the following proposition:

(P) For each ordinal $\alpha \in \Phi$ there is an element $b_{\alpha} \in I$ and an $R$-module $\operatorname{map} \varphi_{\alpha}: \Sigma_{\rho<\alpha} b_{\rho} R \rightarrow R$ such that

(1) If $\beta<\alpha$, then $\varphi_{\alpha}$ restricted to $\sum_{\rho \leqslant \beta} b_{\rho} R$ is $\varphi_{\beta}$,

(2) $\varphi_{\alpha}\left(b_{\alpha}\right) \neq a_{\alpha} b_{\alpha}$.

We prove (P) by transfinite induction. For $\alpha=1$, choose $c_{1} \in J_{1}$ so $c_{1}-a_{1}$ $\notin J$. Then choose $b_{1} \in I$ so $\left(c_{1}-a_{1}\right) b_{1} \neq 0$. Let $\varphi_{1}$ be left multiplication by $c_{1}$.

Now suppose we have proved (P) for all ordinals less than $\delta$. We have a right module homomorphism

$$
\varphi_{\delta}^{\prime}: \sum_{\rho<\delta} b_{\rho} R \rightarrow R,
$$

simply given by the union of the $\varphi_{\rho}, \rho<\delta$. As $R$ is right self-injective, $\varphi_{\delta}^{\prime}$ is given by left multiplication, say by $d_{\delta}$. Let $x$ be an ordinal large enough so $\left\{b_{j}\right\}_{j<\delta} \subset I_{x}$. Choose $c_{\delta} \in J_{x}$ so $c_{\delta}+d_{\delta}-a_{\delta} \notin J$, and then choose $b_{\delta}$ so that $\left(c_{\delta}+d_{\delta}-a_{\delta}\right) b_{\delta} \neq 0$. Define $\varphi_{\delta}$ to be left multiplication by $c_{\delta}+d_{\delta}$. Thus (P) is proved by transfinite induction.

Let $\varphi: \Sigma_{\rho \in \Phi} b_{\rho} R \rightarrow R$ be the right $R$-module map defined by the union of 
the $\varphi_{\rho}$. Then for all $\alpha \in \Phi, \varphi$ restricted to $\Sigma_{\rho<\alpha} b_{\rho} R$ is simply $\varphi_{\alpha}$. Therefore $\varphi\left(b_{\alpha}\right)=\varphi_{\alpha}\left(b_{\alpha}\right) \neq a_{\alpha} b_{\alpha}$; hence, $\varphi$ is not given by left multiplication, contradicting the hypothesis that $R$ is right self-injective. This completes the proof of the theorem.

Proposition 5. Let $A$ be an infinite set. Then there is a totally ordered (by inclusion) subset of the power set of cardinality $2^{|A|}$.

The above proposition allows us to construct the following example. Let $F$ be a countable field and let $A$ be an infinite set of ordinals less than a given cardinality. Let $R_{A}=\prod_{i \in A} F_{i}$ be the direct product of $A$ copies of $F$. Then $R$ is self-injective and $\left|R_{A}\right|=2^{|A|}$. Also, $R_{A}$ has a well-ordered descending chain of annihilators of cardinality $|A|$ and a totally ordered descending chain of annihilators of cardinality $2^{|A|}$. This example shows that 'well ordered' cannot be replaced by 'totally ordered' in the theorem.

THEOREM 6. Let $T$ be a right self-injective ring such that every countable subring is contained in a countable subring $R$, where $T$ is free as a right $R$-module and flat as a left $R$-module. Then $T$ is quasi-Frobenius.

Proof. By Proposition 2 and Theorem 3, it is enough to show that a countable right self-injective ring is quasi-Frobenius.

COROLlary 7. A countable dimensional self-injective algebra over a fiold is quasi-Frobenius.

Corollary 8 (Renault). A group algebra is self-injective only if the group is finite.

Proof. A self-injective group algebra is quasi-Frobenius, hence Artinian, so the group is finite.

Corollary 9. A ring is quasi-Frobenius if and only if every countable subring is contained in a countable self-injective subring.

Proof. This is an easy consequence of Theorems 3 and 4.

If we look at rings without a unity, then most of the above theorems fail to hold. Let $S$ denote the semigroup $\left\{e_{1}, e_{2}, \ldots: e_{i} e_{j}=e_{j}\right\}$. If $F$ is any field, then the semigroup ring $F S$ is left but not right self-injective and is neither right nor left Artinian.

Recall that a twisted group algebra $F^{t} G$ is defined by a 2-cocycle $t: G \times G$ $\rightarrow F-\{0\}$, where $G$ is a group and $F$ is a field, and where we define $\bar{g} \cdot \bar{h}=t(g, h) \overline{g h}$. Define the cocycle subfield of $F$ to be the subfield generated by the image of $t$. Passman has constructed an example of an infinite group such that for certain fields the twisted group algebra is a field. In the same paper [6], Passman proved that if $F$ is algebraically closed and uncountable and $F^{t} G$ is Artinian, then $G$ is finite. We use his idea in the following theorem.

THEOREM 10. Suppose that $F^{t} G$ is a self-injective twisted group algebra such 
that $F$ is a proper extension of the algebraic closure of the cocycle subfield. Then $G$ is finite.

Proof. If $G$ is not finite, then we may assume that it is countably infinite [7], hence $F^{t} G$ is quasi-Frobenius. Let $\Delta(G)$ denote the set of elements in $G$ with finitely many conjugates. Then $F^{t} \Delta(G)$ is self-injective, so $\Delta(G)$ is finite [7]. Now using an argument similar to Passman's [6, p. 648] we may assume that $F^{t} G$ is Artinian and $\Delta(G)=\langle 1\rangle$. Let $K$ denote the cocycle subfield of $F$ and let $L$ denote the algebraic closure of $K$ in $F$. Clearly

$$
F^{t} G \cong F \otimes_{L} L^{t} G
$$

and as $F$ is not algebraic over $L, L^{t} G$ must be an algebraic $L$-algebraic [4]. By a Theorem of Passman, $L^{t} G$ is a semiprime [5, p. 424], so $L^{t} G$ is a semiprime Artinian algebraic algebra over an algebraically closed field. Therefore, $G$ is finite.

\section{REFERENCES}

1. C. Faith, Rings with ascending condition on annihilators, Nagoya Math. J. 27 (1966), 179-191.

2. D. Farkas, Self-injective group algebras, J. Algebra 25 (1973), 313-315.

3. E. Gentile, A note on injective group rings, Proc. Amer. Math. Soc. 23 (1969), 431-432.

4. J. Lawrence, Semilocal group rings and tensor products, Michigan Math. J. 22 (1975), $309-313$.

5. D. Passman, Radicals of twisted group rings, Proc. London Math. Soc. 20 (1970), $409-437$.

6. __ Radicals of twisted group rings. II, Proc. London Math. Soc. 22 (1971), 631-651.

7. A. Reid, Twisted group algebras which are Artinian, perfect or self-injective, Bull. London Math. Soc. 7 (1975), 166-170.

8. G. Renault, Sur les anneaux des groupes, C. R. Acad. Sci. Paris 273 (1971), 84-87.

9. B. Stenstrom, Rings of quotients, Springer-Verlag, New York, 1975.

Department of Mathematics, University of Chicago, Chicago, Illinois 60637

Current address: University of Waterloo, Waterloo, Ontario, Canada N2L 3G1 